\title{
Tagging Two-Photon Production at the LHC
}

\author{
K. Piotrzkowski ${ }^{1}$ \\ CERN, EP Division, CH-1211 Geneva 23, Switzerland \\ and \\ Institute of Nuclear Physics, Kawiory 26A, PL-30055 Kraków, Poland ${ }^{2}$
}

\begin{abstract}
Tagging two-photon production offers a significant extension of the LHC physics programme. Effective luminosity of high-energy $\gamma \gamma$ collisions reaches $1 \%$ of the protonproton luminosity and the standard detector techniques used for measuring very forward proton scattering should allow for a reliable extraction of interesting twophoton interactions. Particularly exciting is a possibility of detecting two-photon exclusive Higgs boson production at the LHC.
\end{abstract}

\section{Introduction}

Two-photon physics has been traditionally studied at $e^{+} e^{-}$colliders owing to large fluxes of virtual photons associated with the beams. However, at the LHC for the first time the proton beam energy will be so high that the effective luminosity of $\gamma \gamma$ collisions will permit performing meaningful and important experiments. The measurements of high-energy $\gamma \gamma$ collisions at the LHC are intrinsically very interesting, and to large extend are complementary to the 'base-line' $p p$ studies.

In this paper, an experimental feasibility of tagging two-photon production in proton-proton collisions at the LHC is considered. The effective $\gamma \gamma$ luminosity of the tagged two-photon collisions is evaluated and used to estimate the physics potential for such measurements.

1 E-mail: krzysztof.piotrzkowski@cern.ch

2 Now at Université catholique de Louvain, FYNU Lab., B-1348 Louvain-la-Neuve 


\section{$2 \quad$ LHC as a $\gamma \gamma$ collider}

For majority of two-photon processes the equivalent photon (or WeizsäckerWilliams) approximation (EPA) can be successfully applied [1]. In EPA twophoton production at the LHC proceeds in two steps: first, two photons are emitted by incoming protons and then, the photons collide producing a system $X$ while the protons remain intact in the elastic production, $p p \rightarrow(\gamma \gamma \rightarrow$ $X) \rightarrow p p X$, or one of them dissociates into a state $N$ in the inelastic production, $p p \rightarrow(\gamma \gamma \rightarrow X) \rightarrow p N X$ 3. Hence, the proton-proton cross-section is a product of the photon-photon cross-section and two photon spectra:

$$
\mathrm{d} \sigma_{p p}=\sigma_{\gamma \gamma} \mathrm{d} N_{1} \mathrm{~d} N_{2}
$$

In EPA the photon spectrum is a function of the photon energy $\omega$ and its virtuality $Q^{2}[1]$ :

$$
\mathrm{d} N=\frac{\alpha}{\pi} \frac{\mathrm{d} \omega}{\omega} \frac{\mathrm{d} Q^{2}}{Q^{2}}\left[\left(1-\frac{\omega}{E}\right)\left(1-\frac{Q_{m i n}^{2}}{Q^{2}}\right) F_{E}+\frac{\omega^{2}}{2 E^{2}} F_{M}\right],
$$

where $\alpha$ is the fine-structure constant, $E$ is the incoming proton energy and the minimum photon virtuality $Q_{\min }^{2} \simeq\left[M_{N}^{2} E /(E-\omega)-M_{p}^{2}\right] \omega / E$, where $M_{p}$ is the proton mass and $M_{N}$ is the invariant mass of the final state $N$. For the elastic production, assuming the dipole approximation for proton form factors, $F_{M}=G_{M}^{2}$ and $F_{E}=\left(4 M_{p}^{2} G_{E}^{2}+Q^{2} G_{M}^{2}\right) /\left(4 M_{p}^{2}+Q^{2}\right)$, and $G_{E}^{2}=G_{M}^{2} / 7.78=$ $\left(1+Q^{2} / 0.71 \mathrm{GeV}^{2}\right)^{-4}$. For the inelastic production $F_{M}=\int \mathrm{d} x F_{2} / x^{3}$ and $F_{E}=$ $\int \mathrm{d} x F_{2} / x$, where $F_{2}\left(x, Q^{2}\right)$ is the proton structure function and $x \simeq Q^{2} / M_{N}^{2}$. The spectrum is strongly peaked at low $\omega$, therefore the photon-photon center of mass energy $W \simeq 2 \sqrt{\omega_{1} \omega_{2}}$ is usually much smaller than the total center of mass energy which equals $2 E=14 \mathrm{TeV}$. For the elastic production the photon virtuality is usually low, $\left\langle Q^{2}\right\rangle \approx 0.01 \mathrm{GeV}^{2}$, therefore the proton scattering angle is very small, $\lesssim 20 \mu \mathrm{rad}$.

The luminosity spectrum of photon-photon collisions, $S_{\gamma \gamma}$, can be introduced in EPA by integration of the product of the photon spectra given by Eq. 1 over the photon virtualities and energies keeping fixed $W$. As an example, in Fig. 1, assuming for each photon an integration interval of $5 \mathrm{GeV}<\omega<E$ and $Q_{\text {min }}^{2}<Q^{2}<2 \mathrm{GeV}^{2}, S_{\gamma \gamma}$ and its integral $\int^{W>W_{0}} d W S_{\gamma \gamma}$ are shown for the elastic production as a function of $W$ and the lower integration limit, $W_{0}$, respectively. The integrated spectrum directly gives a fraction of the $p p$ LHC luminosity available for the photon-photon collisions at $W>W_{0}$. It is remarkable that for $W_{0}=50 \mathrm{GeV}$ this fraction is close to $1 \%$, and for the

$\overline{3 \text { Third }}$ class of events when two protons dissociate is not considered here. 

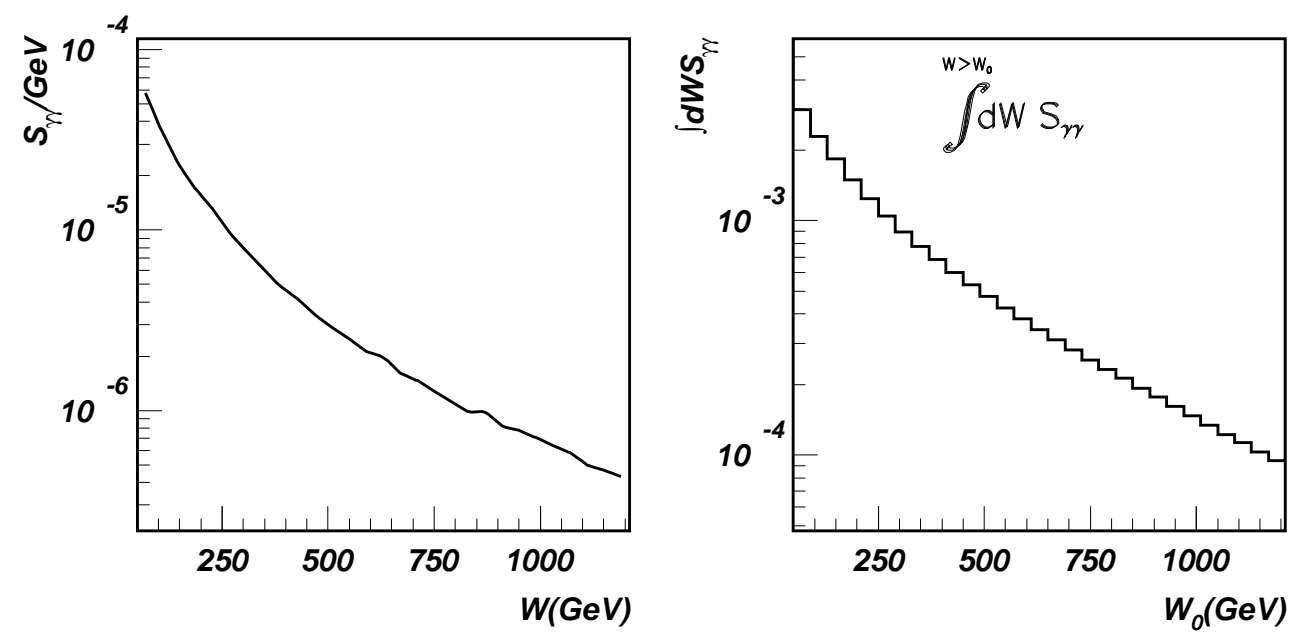

Fig. 1. Elastic $\gamma \gamma$ luminosity spectrum and its integral $\int^{W>W_{0}} d W S_{\gamma \gamma}$ at the LHC, for the range of photon energy and virtuality, $5 \mathrm{GeV}<\omega<7000 \mathrm{GeV}$ and $Q_{\min }^{2}<Q^{2}<2 \mathrm{GeV}^{2}$.

nominal $p p$ luminosity of $10^{34} \mathrm{~cm}^{-2} \mathrm{~s}^{-1}$ the $\gamma \gamma$ luminosity at $W>200 \mathrm{GeV}$ is $3 \times 10^{31} \mathrm{~cm}^{-2} \mathrm{~s}^{-1}$. For the inelastic two-photon production $S_{\gamma \gamma}$ is even larger and will be discussed below.

\section{Tagging two-photon production}

Tagging two-photon production at the LHC would serve two purposes. First, when both elastically scattered protons are detected (double tag) the $\gamma \gamma$ center of mass energy $W$ can be measured independently and improve an overall reconstruction of the final state $X$. Secondly, tagging is needed in order to suppress backgrounds and to obtain a clean two-photon data sample. In particular, the proton scattering angle can be used to extract the two-photon signal both for the double and single tags when only one very forward proton is detected.

At the nominal running conditions the LHC beam at the interaction point (IP) has the Gaussian lateral widths $\sigma_{x}^{*}=\sigma_{y}^{*}=16 \mu \mathrm{m}$ and the angular divergence in horizontal and vertical planes $\sigma_{\theta_{x}}^{*}=\sigma_{\theta_{y}}^{*}=32 \mu \mathrm{rad}$. However, for the initial running at medium luminosity of $10^{33} \mathrm{~cm}^{-2} \mathrm{~s}^{-1}$ almost two times smaller lateral beam size as well as the angular divergence at the IP are expected. At the same time, the event pile-up at the central detectors is not prohibitively large. 
The beam energy spread will be $10^{-4}$ [2]. The beam divergence is comparable with the typical proton scattering angle in the two-photon processes hence effectively protons leave the IP at zero-angle. These protons have however smaller energy than the beam protons and are more strongly deflected in the beam-line magnetic field. The standard method of measuring such forward scattered protons utilizes so-called Roman-pots, position sensitive detectors installed far away from the IP in the beam vacuum to allow the closest possible approach to a beam.

The detector layouts for the Roman-pot detectors so far considered by the Totem [3] and AtLas [4] collaborations, mainly in the context of the total and elastic $p p$ cross-section measurements at the LHC, result in a significant acceptance for zero-angle protons which lost at least a few $\%$ of their initial energy. It would correspond to the tagged photon energies of several hundred $\mathrm{GeV}$ and would therefore limit the studies of two-photon production only to a domain of very large $W$. However, to improve sensitivity to very low angle elastic $p p$ scattering, it was recently proposed [5] to add new detector stations more far away $(\approx 240 \mathrm{~m})$ from the IP. This is also an excellent place for tagging two-photon production since at this location, for the nominal LHC beam optics in the horizontal plane, the so-called betatron phase advance is $\approx \pi$, the beam size has a minimum, and the dispersion $D$ is large, about $100 \mathrm{~mm}$. It means that in the horizontal plane a detector can approach the beam very closely and at the same time the average horizontal displacement $\Delta x$ with respect to the beam axis due to the proton energy loss is large, $\Delta x=D \omega / E$. Therefore, measurement of $\Delta x$ gives directly the tagged photon energy. Additionally, the angle between the proton momentum and the beam axis, $\theta_{x}$, is proportional to the same angle at the IP, $\theta_{x} \simeq \theta_{x}^{*} / 3$. On the other hand, in the vertical plane the betatron phase advance is $\approx \pi / 2$ at this location, therefore the angle $\theta_{y}$ is proportional to the proton vertical displacement at the IP and the vertical proton displacement $\Delta y[\mathrm{~mm}] \simeq 0.01 \times \theta_{y}^{*}[\mu \mathrm{rad}]$. Hence, the measurement of $\theta_{x}$ and $\Delta y$ directly provide information on proton transverse momentum at the IP, $p_{T}$, hence on photon virtuality $Q^{2} \simeq p_{T}^{2} \simeq E^{2}\left(\theta_{x}^{* 2}+\theta_{y}^{* 2}\right)$.

To ensure enough room for the beam steering and to keep the detectors in a 'shadow' of beam collimators a $1 \mathrm{~mm}$ minimum distance between the detector edge and the beam axis should be assumed [3]. Since the proton beam size in the horizontal plane is small, $\sigma_{x} \simeq 3 \sigma_{x}^{*} \lesssim 50 \mu \mathrm{m}$, this distance corresponds to more than 20 beam widths, far away from the beam core. The minimum approach of $1 \mathrm{~mm}$ corresponds to the minimum tagged photon energy of $70 \mathrm{GeV}$. The maximum tagged energy is about $700 \mathrm{GeV}$ due to a beam-line geometrical acceptance, and because for such a large energy loss $D$ starts to change with the scattered proton energy, making the energy measurement less reliable. Assuming this tagged energy range, the photon-photon luminosity spectrum is shown in Fig. 2, for the double tagging, when both scattered protons are detected as well as for the single tagging, when at least one scattered proton 

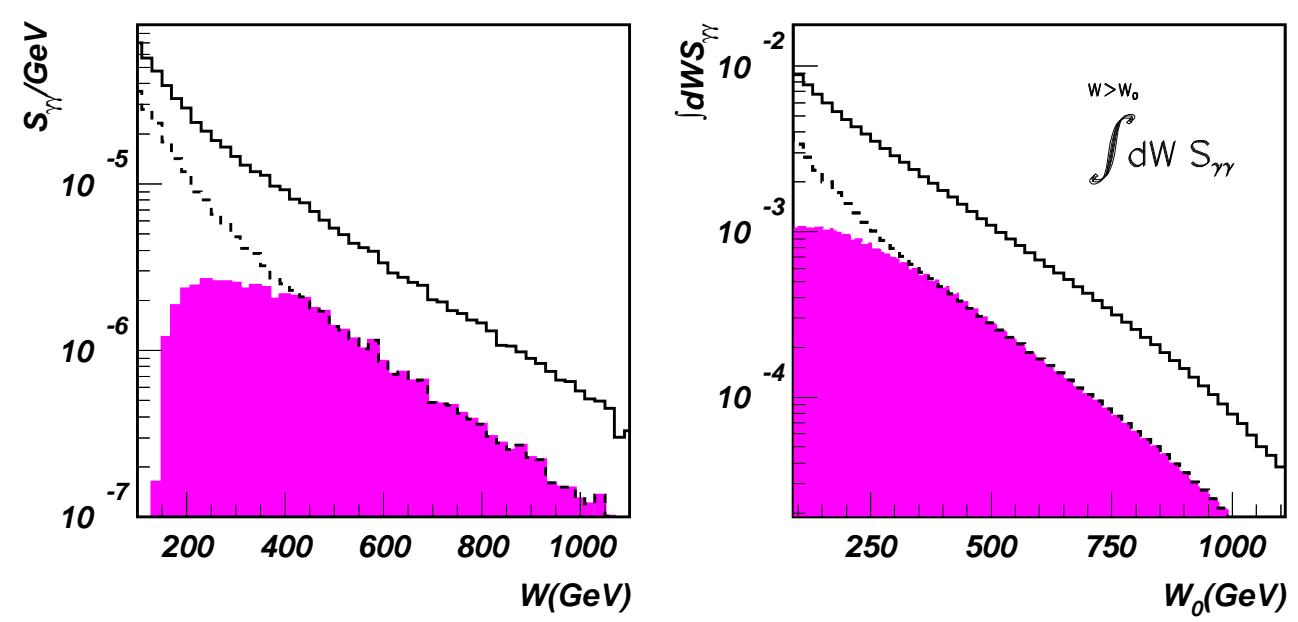

Fig. 2. Tagged photon-photon luminosity spectrum $S_{\gamma \gamma}$ and its integral $\int^{W>W_{0}} d W S_{\gamma \gamma}$ assuming double tags (shaded histograms) and single tags, for all (solid line) and only for elastic (dashed line) events; the tagging range is $70<\omega<700 \mathrm{GeV}$ and $Q_{\min }^{2}<Q^{2}<2 \mathrm{GeV}^{2}$.

is detected; photon virtuality is restricted to $Q_{\min }^{2}<Q^{2}<2 \mathrm{GeV}^{2}$. It can be noted that the $\gamma \gamma$ luminosity spectrum for the double tagging is sizable in the range $200 \lesssim W \lesssim 500 \mathrm{GeV}$, whereas the single tagging preserves a major fraction of the total elastic $\gamma \gamma$ luminosity. Including the inelastic contribution to the single tagged spectrum increases $S_{\gamma \gamma}$ approximately by a factor of three, assuming the maximum dissociative mass $M_{N}$ of $20 \mathrm{GeV} 4$.

The required detector size is small, with a sensitive area of each detector plane of the order of $2 \mathrm{~cm}^{2}$, with excellent spatial resolution in the 10-20 $\mu \mathrm{m}$ range. There should be two detector stations separated by $2-4 \mathrm{~m}$ to ensure a precise measurement of direction of the proton momentum. Modern silicon micro-strip or pixel sensors are the most probable candidates. The crucial alignment of the detector sensors with respect to the beam axis should be possible using the elastic $p p$ events when protons cross several detector planes. Setting the final momentum scale requires a precise knowledge of the integral of the magnetic field along the scattered proton trajectory, however the photon energy scale might be set using the data where the final state $X$ is fully detected in the central detectors and a precise and independent determination of $W$ is possible.

For the above spatial resolutions, the final resolution on $W$ and photon virtu-

$\overline{4}$ This ensures that debris of the system $N$ are not observed in the central detectors. 
alities would be determined by the geometrical beam properties at the IP. In this case, the horizontal displacement due proton energy loss $\Delta x$ is smeared by $\sigma_{x} \lesssim 50 \mu$ m leading to a $5 \mathrm{GeV}$ uncertainty on $W=200 \mathrm{GeV}$, for example.

The same reaction, $p p \rightarrow p p X$, occurs also in strong interactions, via fusion of two colorless objects, pomerons, and will therefore interfere with the two-photon fusion. However, this so-called central diffraction usually results in much larger transverse momenta of the scattered protons, following the distribution $\exp \left(-b p_{T}^{2}\right)$, with the expected at the LHC diffractive slope $b \simeq 4 \mathrm{GeV}^{-2}$ [6]. Soft pomeron-pomeron interactions have several orders of magnitude larger cross-sections than the $\gamma \gamma$ interactions, but for the hard processes the cross-sections are of similar size (see below). Therefore, the measurement of proton $p_{T}$ is vital for extracting the $\gamma \gamma$ signal. In Fig. 3, the 'true' and smeared distributions of $p_{T}^{2}$ are compared assuming the same cross-sections integrated over $p_{T}^{2}$. It shows that in such a case the two-photon signal is clearly visible and can be well extracted, and for example, for $p_{T}^{2}<0.05 \mathrm{GeV}^{2}$ the pomeron-pomeron contribution (neglecting interference effects) is about $20 \%$. One should note that for the double tagged events the separation is even more powerful since one can independently use for that purpose $p_{T}$ of each proton.
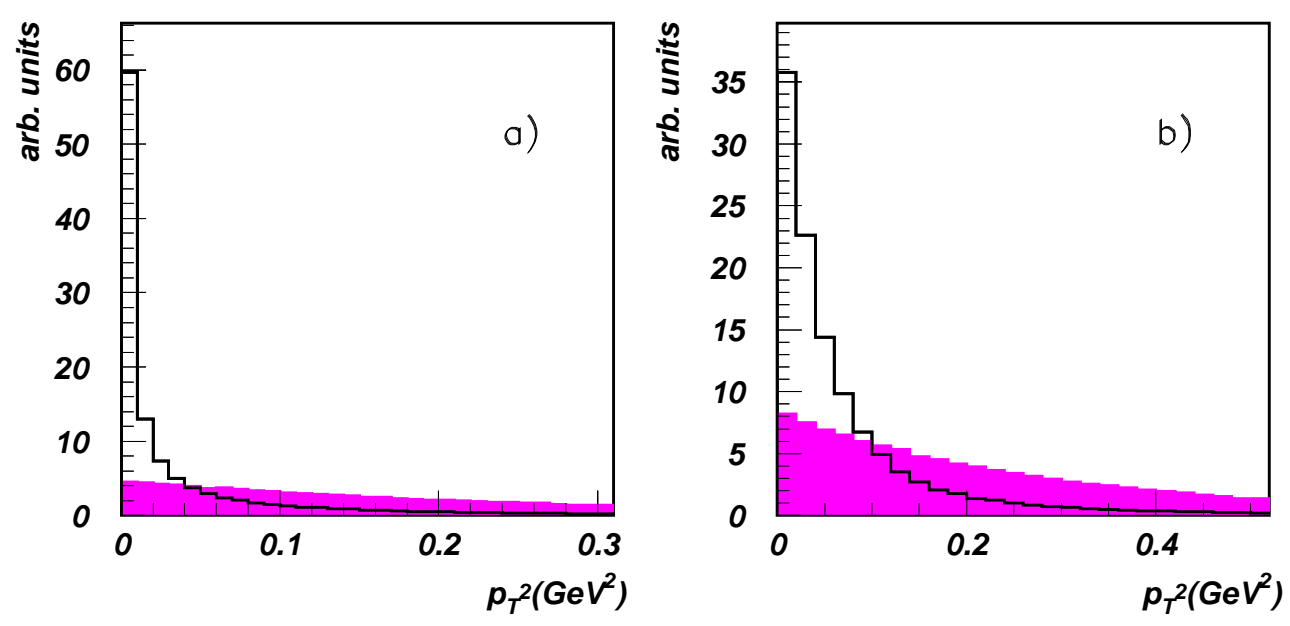

Fig. 3. a) Distribution of the transverse momenta squared of the scattered protons for the two-photon (empty histogram) and pomeron-pomeron (shaded histogram) collisions assuming the diffractive slope $b=4 \mathrm{GeV}^{-2}$; b) the same distributions smeared by the beam divergence for the initial running conditions. Distributions have the same normalization for $p_{T}^{2}<2 \mathrm{GeV}^{2}$ and correspond to a $100 \mathrm{GeV}$ proton energy loss. 


\section{Examples of tagged two-photon physics potential}

The exclusive $\gamma \gamma$ production of one or two heavy particles, as for example in $\gamma \gamma \rightarrow H, t \bar{t}$, or $W^{+} W^{-}$processes, is particularly interesting. These events are clean - two (or one) very forward protons measured far away from the IP and only one or two particles produced and decaying in the central detectors, giving an additional handle for background suppression.

In the exclusive two-photon Higgs boson production in leading order the photons couple via a fermion loop which results in sensitivity to any new fermion state, even significantly beyond the $W$ scale, hence to possible departures from the Standard Model (SM) physics.
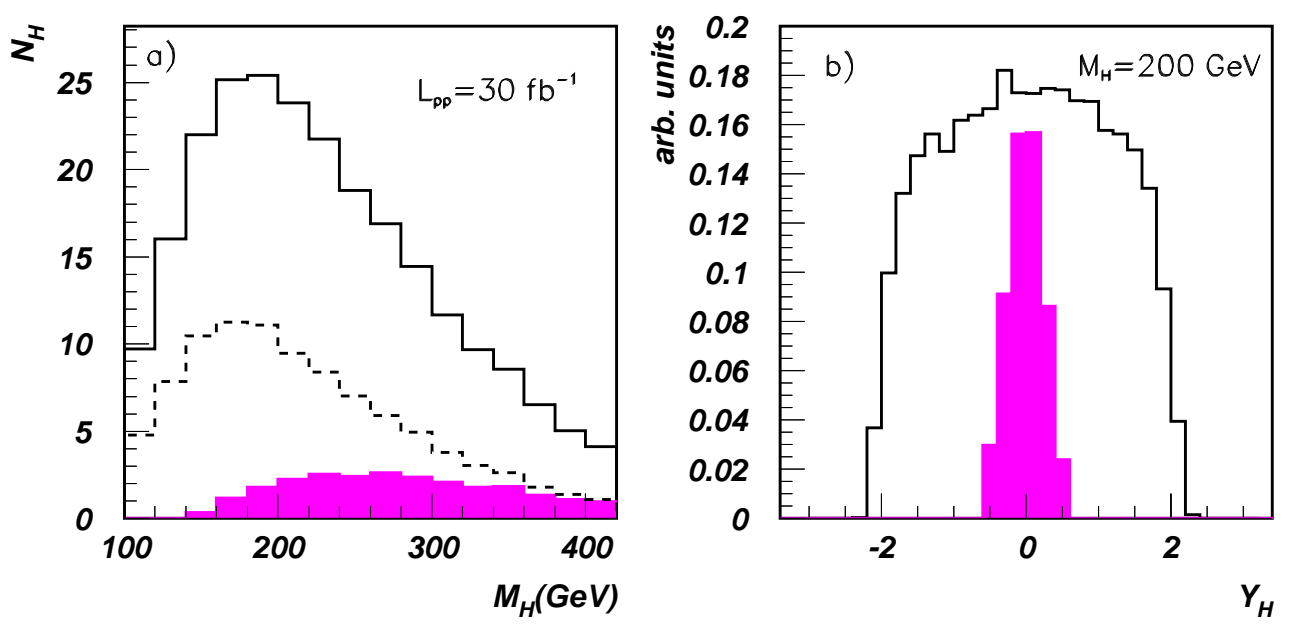

Fig. 4. a) Number of the SM Higgs boson events as a function of its mass, exclusively produced in $\gamma \gamma$ collisions for the integrated $p p$ luminosity of $30 \mathrm{fb}^{-1}$, assuming double tags (shaded histograms), and single tags for all (solid line) and only for elastic (dashed line) scattering; b) Rapidity distribution for the tagged Higgs boson production.

The number $N_{H}$ of the two-photon produced Higgs bosons is given by [7]:

$$
N_{H}=\frac{4 \pi^{2} \Gamma_{\gamma \gamma}}{M_{H}^{2}} L_{p p} S_{\gamma \gamma}\left(W=M_{H}\right)
$$

where $L_{p p}$ is the proton-proton integrated luminosity, $M_{H}$ is the Higgs boson mass and $\Gamma_{\gamma \gamma}$ is the $H \rightarrow \gamma \gamma$ width. In Fig. $4, N_{H}$ expected in SM is shown for the integrated $p p$ luminosity which corresponds to first three years of the LHC running at medium luminosity. It shows that the double-tagged Higgs 
boson production is statistically very limited at low $M_{H}$, but the single-tagged production is not negligible starting already at $M_{H}=100 \mathrm{GeV}$.

For the same luminosity more than five thousand 'gold-plated' double-tagged $W^{+} W^{-}$pairs would be exclusively produced at $W>200 \mathrm{GeV}$, assuming the asymptotic value of $\sigma_{\gamma \gamma \rightarrow W W} \simeq 200 \mathrm{pb}$. The corresponding number of the exclusive top pairs is unfortunately more than hundred times smaller. The exclusive $W^{+} W^{-}$production constitutes also an 'irreducible' background for the events when Higgs boson decays into $W^{+} W^{-}$. Therefore, for $M_{H} \gtrsim 200 \mathrm{GeV}$ the signal $H \rightarrow Z Z$ seem experimentally more preferable. Given significant backgrounds and low signal statistics, such two-photon measurements cannot possibly be used for a Higgs boson search, but they would provide an important handle on $\Gamma_{\gamma \gamma}$ at the LHC [8].

As was mentioned before, the same event topology have also the pomeronpomeron interactions. Recent studies [6] show that the pomeron-pomeron exclusive Higgs boson production has a similar cross-section to the two-photon case, therefore the two-photon signal can be statistically extracted using the distributions of the proton $p_{T}$. The pomeron-pomeron cross-section is theoretically not well controlled due to soft final-state interactions and the associated survival probability. In contrast, provided that $Q^{2}$ is not too large, the twophoton cross-section is much less sensitive to these effects [6]. On the other hand, interference between the pomeron-pomeron and $\gamma \gamma$ amplitudes might give information (in analogy to Coulomb and elastic $p p$ scattering, for example) about the amplitude behaviour at energies beyond available at the LHC.

Of course, the photon-photon measurements would also extend search for the new physics at the LHC. In particular, $\gamma \gamma$ search for new particles expected in supersymmetric models would be to large extend complementary to the corresponding proton-proton studies [9].

Many interesting QCD studies should also be possible, as for example measurements of the exclusive production of multi-jets with large transverse energy, or the vector meson and photon production at very high transverse momenta [10].

\section{Summary}

The initial studies presented in this paper indicate that the installation at about $240 \mathrm{~m}$ from the IP of the recently proposed detectors might permit, at relatively low cost and effort, to utilize the LHC as a high-energy $\gamma \gamma$ collider. The significant luminosity of the tagged photon-photon collisions open in particular an exciting possibility of studying the exclusive production of the Higgs boson, and for searches of new phenomena in high-energy $\gamma \gamma$ collisions. 


\section{Acknowledgements}

I would like to thank Vincent Lemaitre for very useful discussions and comments.

\section{References}

[1] V.M. Budnev et al., Phys. Reports 15 (1975) 181.

[2] LHC Conceptual Design Report, CERN/AC/95-05.

[3] TOTEM Collab., Technical Proposal, CERN/LHCC/99-07.

[4] ATLAS Collab., Technical Design Report, CERN/LHCC/99-01.

[5] A. Faus-Golfe et al., Precise measurement of the total cross-section and the Coulomb scattering at the LHC, Proc. of EPAC2000 Conf. at Vienna, p. 352.

[6] V.A. Khoze et al., Eur. Phys. Journal C 14 (2000) 525.

[7] D.L. Borden et al., Phys. Rev. D 48 (1993) 4018.

[8] E. Papageorgiu, Phys. Lett. B 352 (1995) 394.

[9] J. Ohnemus et al., Phys. Lett. B 328 (1994) 369.

[10] N.G. Evanson and J.R. Forshaw, Phys. Rev. D 60 (1999) 034016. 